\title{
The effects and mechanisms of the action of galangin on spatial memory in rats
}

\author{
Kilic FS ${ }^{1}$, Kaygisiz B ${ }^{1}$, Aydin $\mathrm{S}^{1}$, Yildirim $\mathrm{E}^{1}$, Oner $\mathrm{S}^{2}$, Erol $\mathrm{K}^{1}$ \\ Eskisehir Osmangazi University Medical School, Department of Pharmacology, Eskisehir, Turkey. \\ fskilic@ogu.edu.tr
}

\begin{abstract}
BACKGROUND: Galangin, a flavonoid compound with acetylcholinesterase inhibitory activity, may improve cognitive functions by enhancing cholinergic transmission.

OBJECTIVES: We aimed to investigate the effects of galangin on spatial memory impairment in rats. METHODS: The effects of galangin (50 and $100 \mathrm{mg} / \mathrm{kg}$ ) and reference anti-dementia drug donepezil (1mg/ $\mathrm{kg}$ ) administrations were examined on memory impairment induced by the muscarinic cholinergic receptor antagonist scopolamine or the nicotinic cholinergic receptor antagonist mecamylamine in the Morris water maze (MWM) test. Hippocampal acetylcholine concentrations were also determined.

RESULTS: Galangin 50 and $100 \mathrm{mg} / \mathrm{kg}$ significantly decreased the mean distance to platform and increased the time spent in the escape platform quadrant in scopolamine-treated rats. Galangin $100 \mathrm{mg} / \mathrm{kg}$ significantly decreased the mean distance to platform and increased the time spent in the escape platform quadrant in mecamylamine-treated rats. The effects of galangin in the MWM were comparable with donepezil. Scopolamine and mecamylamine decreased acetylcholine concentrations, whereas galangin both alone and with mecamylamine or scopolamine administration increased acetylcholine concentrations.

CONCLUSION: Galangin improved memory impairment comparable to donepezil and nicotinic and muscarinic receptors may be involved in this effect. Galangin may be considered as a promising flavonoid in the prevention and treatment of memory impairment in Alzheimer's disease and other dementias (Fig. 7, Ref. 37). Text in PDF www.elis.sk. KEY WORDS: spatial memory, cognition, galangin, Morris water maze, rats.
\end{abstract}

\section{Introduction}

It is estimated that there are 24.3 million people with dementia worldwide and this number will be about 81.1 million by 2040 . Alzheimer's disease is the most frequent cause of dementia, followed by vascular disease $(1,2)$. Dementia is characterized by a decline in cognitive functions, primarily in learning and memory, behavioral alterations, and decreased daily functioning (3). The late stages of the disease bring deteriorations in spatial functions and self-management. It was shown that the hippocampal region was associated with spatial learning and memory in the brain (4). This region has a unique role in memory functions by connecting different components of experiences about life (5).

The cholinergic system, with its complicated network in the central nervous system is essential for maintaining cognitive,

${ }^{1}$ Eskisehir Osmangazi University Medical School, Department of Pharmacology, Eskisehir, Turkey, and ${ }^{2}$ Eskisehir Osmangazi University Medical School, Department of Biostatistics, Eskisehir, Turkey

Address for correspondence: F.S. Kilic, MD, Eskisehir Osmangazi University Medical School, Department of Pharmacology, Meselik Kampusu, 26480, Eskisehir, Turkey.

Phone: +90.222 .2392979 , Fax: +90.222 .2393772$

Acknowledgement: This study was supported by the Scientific Research Projects Commission of Eskisehir Osmangazi University (Project number: 201393). emotional, and behavioral functions normally (6). In addition, this system is one of the most important neurotransmitter systems functioning in spatial learning and memory (7). The functions of cholinergic system are mediated by acetylcholine through muscarinic and nicotinic receptors (8).

Cognitive impairments are associated with many neurodegenerative diseases such as: Alzheimer's and Parkinson's disease (9). Enhancing cholinergic transmission in the brain produces improvements in cognitive functions, especially in learning and memory. Inhibition of acetylcholinesterase, which catalyzes the degradation of acetylcholine, is one of the strategies for enhancing cholinergic transmission (10). Currently, acetylcholinesterase inhibitors donepezil, galantamine, and rivastigmine are approved and commonly used for symptomatic treatment of cognitive dysfunctions in Alzheimer's disease and other dementias (11, 12). Researchers are focused on developing new agents with a cholinesterase-inhibiting effect. Galangin (3,5,7-trihydroxyflavone) is a flavonoid found in Alpinia officinarum. Flavonoids are phytochemicals beneficial for human health, which play significant roles in various biologic processes and have been center of interest in scientific researches $(13,14)$. The antiproliferative, anticancer, and anti-inflammatory features of galangin have been reported $(15,16)$. In addition, the acetylcholinesterase-inhibiting activity of galangin was reported with the highest inhibitory activity among studied 21 flavonoids (17). 


\section{1-886}

In this study, we aimed to investigate the effects of acute galangin administration on spatial memory and mechanisms involved in rats in the Morris water maze (MWM) test. The MWM is a test used to investigate spatial learning and memory, it is superior to other tests that assess learning and memory $(18,19)$. For this purpose, galangin was administered both alone and with a nicotinic cholinergic receptor antagonist, mecamylamine or with a muscarinic cholinergic receptor antagonist, scopolamine. In addition, the effects of galangin on hippocampal acetylcholine concentrations were also studied. The effects of galangin in the MWM were compared to donepezil, which was used as a reference anti-dementia drug.

\section{Material and methods}

\section{Experimental Design and Groups}

The effects of acute galangin administration were investigated on mecamylamine or scopolamine-induced memory impairment using a spatial navigation task in the MWM in rats. Mecamylamine, a nicotinic cholinergic receptor antagonist or scopolamine, a muscarinic cholinergic receptor antagonist, were used to induce memory impairment and also to assess possible involving muscarinic or nicotinic cholinergic mechanisms in the effects of galangin. To compare the effects of galangin in MWM, donepezil was used as a reference drug, which has acetylcholinesterase inhibitor activity and is approved for Alzheimer's disease and other dementias (20). The groups were composed as below:

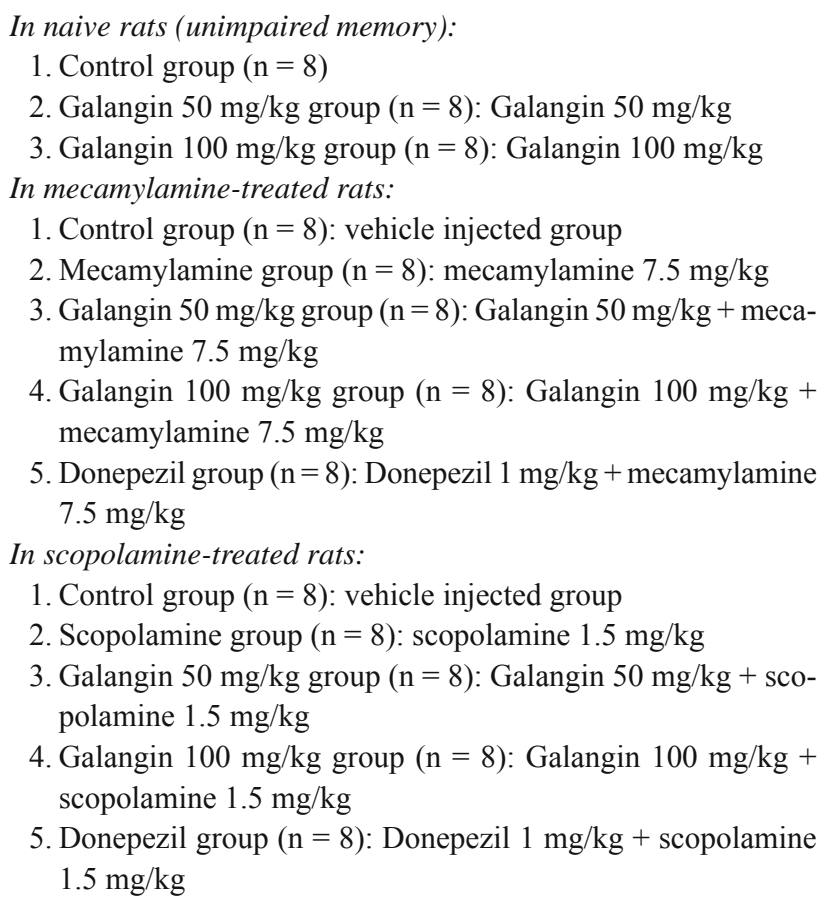

Acute galangin or donepezil administrations were performed in the retention test. Mecamylamine or scopolamine were administered 30 minutes after galangin or donepezil injections and then the rats were immediately released to the pool for the retention test.

\section{Animals}

Male Sprague Dawley rats (250-300 g) were used in the study. Animals were sheltered in standard conditions of light (12 hours light / dark cycle) and temperature $\left(21 \pm 1{ }^{\circ} \mathrm{C}\right)$. Food and water were available ad libitum. All the experiments were performed with the permission of the Local Ethics Committee of Eskisehir Osmangazi University for the care and use of laboratory animals (Date and number of the permission: 27-02-2013 / 317).

Drugs

Galangin (Alfa Aesar) were dissolved in distilled water: DMSO (5:1 ratio) and administered intraperitoneally. Donepezil hydrochloride monohydrate (Sigma-Aldrich) and mecamylamine hydrochloride (Santa Cruz Biotechnology) were dissolved in saline and injected subcutaneously. Scopolamine hydrobromide trihydrate (Acros Organics) were dissolved in saline and given intraperitoneally.

\section{Morris Water Maze (MWM)}

The MWM is the test used for evaluating spatial learning and memory (19). Test was performed according to literature (21). Time spent in the escape platform quadrant (s) and the mean distance to platform $(\mathrm{cm})$ were recorded and analyzed using a video tracking system (Noldus Ethovision XT, Version 9, Wageningen, The Netherlands). The "mean distance to platform" means "the mean of the distance to the quadrant, in which the platform is positioned during a 60 -s probe trial performance". If this value was smaller, it means that the rat swam closer to the escape platform during the probe test". The time spent in the escape platform quadrant and the mean distance to the platform calculations were used as measures for the development of spatial memory.

\section{Determination of hippocampal acetylcholine concentrations}

After the retention test, rats were sacrificed immediately and their hippocampal regions were dissected out for the determination of acetylcholine concentrations. Tissues were stored at $-80^{\circ} \mathrm{C}$ until the determination of acetylcholine concentrations. Acetylcholine concentrations were determined using an enzyme-linked immunosorbent assay, which was performed in accordance with the instructions of the commercial kit (EnzyChromTM Acetylcholine Assay Kit (EACL-100), BioAssay Systems, CA, USA).

\section{Statistical analysis}

The SPSS Version 21 program was used for a statistical analysis. Data were statistically analyzed using one-way analysis of variance (ANOVA) or the Kruskal-Wallis test. In addition, the Tamhane test was used for multiple comparisons. $\mathrm{P}<0.05$ was accepted as statistically significant.

\section{Results}

The effects of galangin on unimpaired memory in naive rats in MWM

Galangin at both doses, 50 and $100 \mathrm{mg} / \mathrm{kg}$, did not alter the time spent in the escape platform quadrant and the mean distance to 


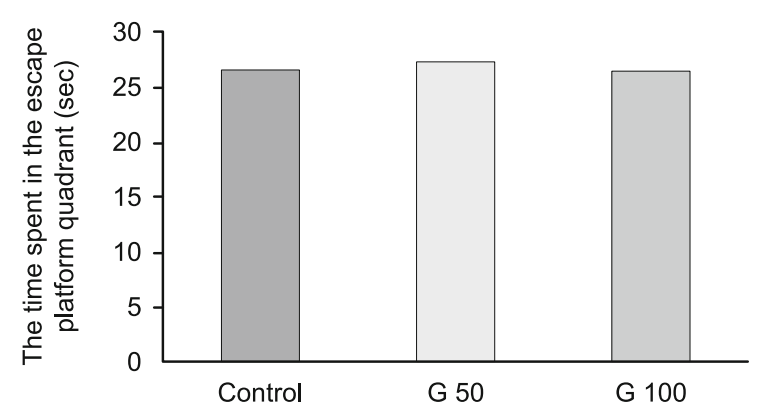

Fig. 1. The effects of galangin on the time spent in the escape platform quadrant in naïve rats. Results are given as the median (25-75 $\%$ percentiles). G 50: Galangin $50 \mathrm{mg} / \mathrm{kg}, \mathrm{G} 100$ : Galangin $100 \mathrm{mg} / \mathrm{kg}$.

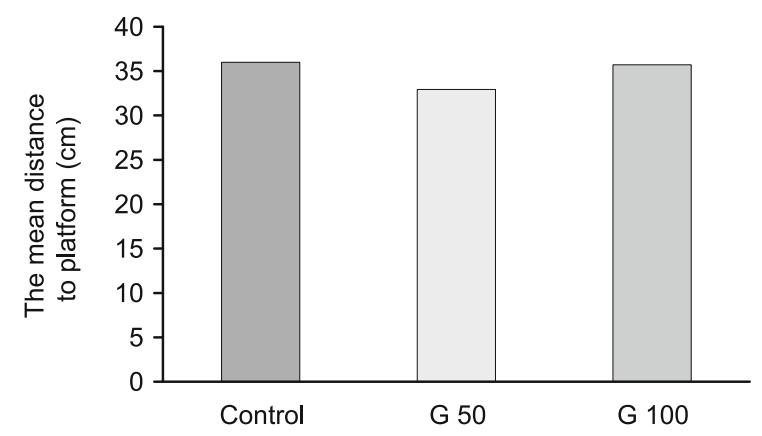

Fig. 2. The effects of galangin on the mean distance to platform in naïve rats. Results are given as the median ( $25-75 \%$ percentiles). G 50: Galangin $50 \mathrm{mg} / \mathrm{kg}, \mathrm{G}$ 100: Galangin $100 \mathrm{mg} / \mathrm{kg}$.

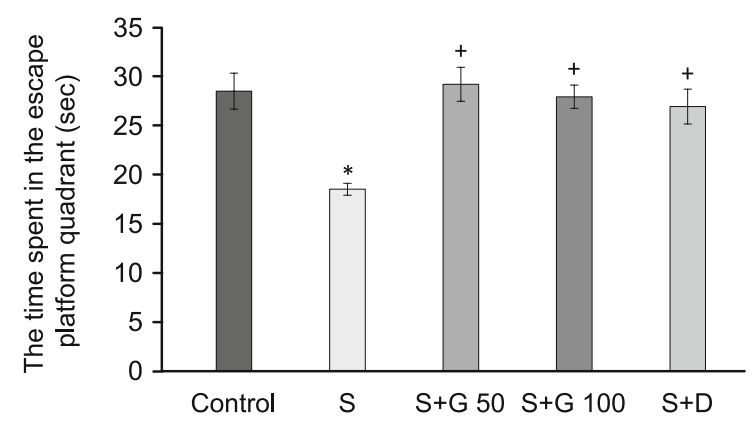

Fig. 3. The effects of galangin on the time spent in escape platform quadrant in rats with scopolamine-induced memory impairment. Results are given as the mean \pm SEM. * $p<0.05$, compared with the control; $+p<0.05$, compared with the scopolamine group. $S$ : Scopolamine, S+G 50: Scopolamine+Galangin $50 \mathrm{mg} / \mathrm{kg}, \mathrm{S}+\mathrm{G} 100$ : Scopolamine+Galangin $100 \mathrm{mg} / \mathrm{kg}, \mathrm{S}+\mathrm{D}$ : Scopolamine+Donepezil.

platform compared to the control, when it was administered alone without scopolamine or mecamylamine, which induce memory impairment in rats $(\mathrm{p}>0.05)$ (Figs 1 and 2, respectively).

The effects of galangin on scopolamine-induced memory impairment in MWM

There was a significant decrease in the time spent in the escape platform quadrant and a significant increase in the mean distance to platform in scopolamine alone-injected rats compared to the control group. Galangin at both doses of 50 and $100 \mathrm{mg} / \mathrm{kg}$ and donepezil $1 \mathrm{mg} / \mathrm{kg}$ significantly increased the time spent in the escape platform quadrant and decreased the mean distance to platform in scopolamine-injected rats compared to scopolamine alone-injected rats $(\mathrm{p}<0.05)$ (Figs 3 and 4, respectively).

The effects of galangin on mecamylamine-induced memory impairment in MWM

There was a significant decrease in the time spent in the escape platform quadrant and a significant increase in the mean distance to platform in mecamylamine alone-injected rats compared to the control group. Galangin at the $100 \mathrm{mg} / \mathrm{kg}$ dose and donepezil 1 $\mathrm{mg} / \mathrm{kg}$ significantly increased the time spent in the escape platform quadrant and decreased the mean distance to platform in mecamylamine-injected rats compared to mecamylamine aloneinjected rats $(\mathrm{p}<0.05)$ (Figs 5 and 6, respectively).

The effects of galangin on hippocampal acetylcholine concentrations in rats

Scopolamine or mecamylamine significantly decreased acetylcholine concentrations compared to the control (Fig. 7). Both

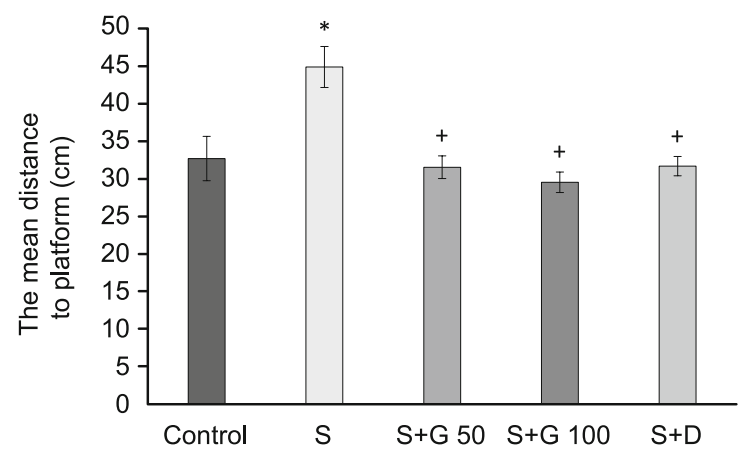

Fig. 4. The effects of galangin on the mean distance to platform in rats with scopolamine-induced memory impairment. Results are given as the mean \pm SEM. * $\mathbf{p}<0.05$, compared to the control; $+\mathbf{p}<$ 0.05 , compared to the scopolamine group. S: Scopolamine, $S+G$ 50: Scopolamine + Galangin $50 \mathrm{mg} / \mathrm{kg}$, S+G 100: Scopolamine + Galangin $100 \mathrm{mg} / \mathrm{kg}, \mathrm{S}+\mathrm{D}$ : Scopolamine+Donepezil.

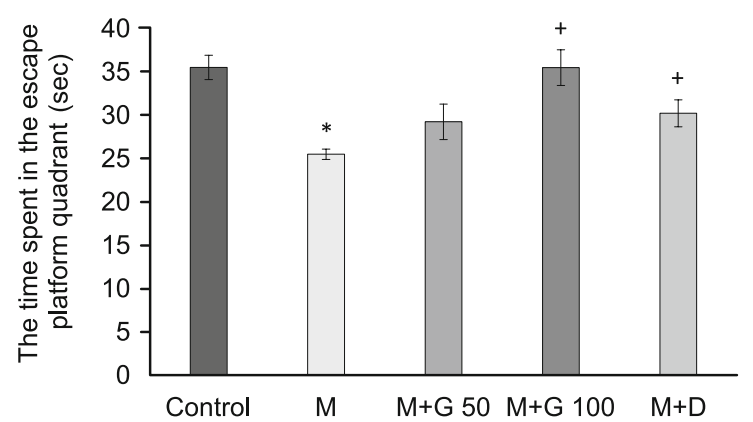

Fig. 5. The effects of galangin on the time spent in the escape platform quadrant in rats with mecamylamine-induced memory impairment. Results are given as the mean \pm SEM. * $\mathbf{p}<0.05$, compared to control; + $\mathbf{p}<\mathbf{0 . 0 5}$, compared to mecamylamine group. M: Mecamylamine, $\mathrm{M}+\mathrm{G}$ 50: Mecamylamine+Galangin $50 \mathrm{mg} / \mathrm{kg}, \mathrm{M}+\mathrm{G}$ 100: Mecamylamine + Galangin 100 mg/kg, M+ D: Mecamylamine +Donepezil. 


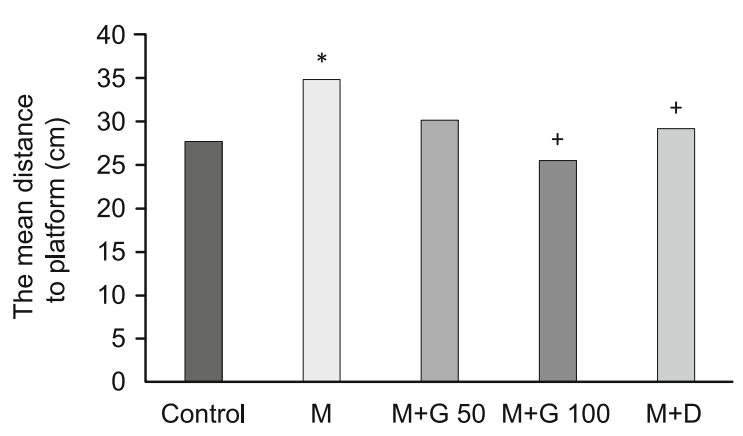

Fig. 6. The effects of galangin on the mean distance to platform in rats with mecamylamine-induced memory impairment. Results are given as the median $(25-75 \%$ percentiles). * $p<0.05$, compared to the control; $+p<0.05$, compared to the mecamylamine group. M: Mecamylamine, M+G 50: Mecamylamine + Galangin $50 \mathrm{mg} / \mathrm{kg}, \mathrm{M}+\mathrm{G}$ 100: Mecamylamine+Galangin $100 \mathrm{mg} / \mathrm{kg}, \mathrm{M}+\mathrm{D}$ : Mecamylamine+Donepezil.

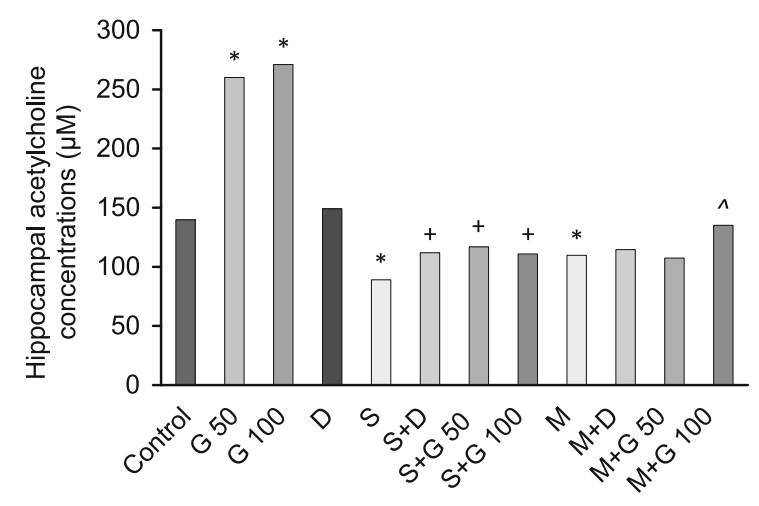

Fig. 7. The effects of galangin alone or in combination with scopolamine or mecamylamine on hippocampal acetylcholine concentrations. G 50: Galangin $50 \mathrm{mg} / \mathrm{kg}$, G 100: Galangin $100 \mathrm{mg} / \mathrm{kg}$, S: scopolamine, $\mathrm{M}$ : mecamylamine, $S+G$ 50: Scopolamine+Galangin $50 \mathrm{mg} / \mathrm{kg}, \mathrm{S}+\mathrm{G} 100$ : Scopolamine + Galangin $100 \mathrm{mg} / \mathrm{kg}, \mathbf{M}+\mathbf{G} 50$ : Mecamylamine + Galangin $50 \mathrm{mg} / \mathrm{kg}, \mathrm{M}+\mathrm{G}$ 100: Mecamylamine+Galangin $100 \mathrm{mg} / \mathrm{kg}$. * $\mathrm{p}<0.05$, compared to the control, $+p<0.05$, compared to scopolamine ${ }^{\wedge}$ compared to the mecamylamine group.

galangin doses increased acetylcholine concentrations, when administered with scopolamine compared to the scopolamine alone group (Fig. 7). In addition, galangin $100 \mathrm{mg} / \mathrm{kg}$ dose increased acetylcholine concentrations, when administered with mecamylamine, compared to mecamylamine alone group (Fig. 7). Also, galangin at both doses of 50 and $100 \mathrm{mg} / \mathrm{kg}$ increased acetylcholine concentrations compared to the control in treatment-naïve rats that did not take scopolamine or mecamylamine injections (Fig. 7). In this aspect, we may suggest that galangin may be preventive for memory impairment.

\section{Discussion}

In this study, we investigated the effects of acute galangin administration on scopolamine or mecamylamine-induced memory impairment in the MWM in rats. We observed that galangin prevented memory impairment induced by both scopolamine and mecamylamine. These effects were comparable to the reference drug donepezil, which is used in the treatment of dementia disorders. In addition, galangin increased hippocampal acetylcholine concentrations, when administered alone and also prevented the decrease in hippocampal acetylcholine concentrations induced by scopolamine or mecamylamine.

Alzheimer's disease is one of the most common causes of dementia (22). Although, there are acetylcholinesterase inhibitors that are used for the treatment of cognitive dysfunction in dementia, researchers are focused on developing more effective acetylcholinesterase inhibitors (23).

Galangin is a flavonoid compound that is commonly used in traditional medicine (24). Many bioactivities of galangin such as: antiinflammatory, anticancer, and antiviral effects have been reported (25). In addition, based on the studies reporting the potent acetylcholinesterase inhibitory effect of galangin, it was suggested that galangin might be a potential treatment option in Alzheimer's disease and other dementias $(17,23,26,27)$. Low brain acetylcholine concentrations, especially in the hippocampus, are associated with cognitive dysfunction and increasing acetylcholine concentrations in the brain via inhibition of acetylcholinesterase, which is accepted as a helpful way of treating the cognitive dysfunction seen in Alzheimer's disease and other dementias (28, 29). In this study, galangin prevented the memory impairment induced by scopolamine or mecamylamine. These results may suggest a potential role for galangin in the treatment of dementia. Moreover, a preventive role for galangin may be suggested in memory impairment considering its enhancing effect on hippocampal acetylcholine concentrations, when administered alone. In contrast to our results, galangin showed no beneficial effect on D-galactose-induced cognitive dysfunction in mice (30). However, this study differs from our study in terms of the research method and the animals. In our study, we induced memory impairment by administering the muscarinic acetylcholine receptor antagonist scopolamine or nicotinic acetylcholine receptor antagonist mecamylamine to rats. Scopolamine is reported to inhibit cholinergic functions in the central nervous system and increase the activity of acetylcholinesterase $(31,32)$.

Galangin prevented both scopolamine and mecamylamineinduced memory impairment. Thus, we may suggest that galangin exerted this effect by affecting muscarinic and nicotinic acetylcholine receptors. This dual effect of galangin on both acetylcholine receptors may be explained by the increased acetylcholine concentrations in the hippocampus. We observed that acute galangin administration increased hippocampal acetylcholine concentrations at both doses of 50 and $100 \mathrm{mg} / \mathrm{kg}$, when administered alone without scopolamine or mecamylamine injections. This is in accordance with the reported acetylcholinesterase inhibitory effect of galangin (17). In addition, galangin increased hippocampal acetylcholine concentrations, when administered in combination with scopolamine or mecamylamine. Moreover, it was reported that galangin exerted a neuroprotective effect in an Alzheimer's disease model and researchers suggested a potential role for galangin in its treatment (33). Additionally, it was observed that galangin inhibited 
experimentally induced neuroinflammation and considered it as a candidate agent in the treatment of neuroinflammatory diseases such as: Alzheimer's and Parkinson's disease (34). These studies present data supporting our results showing the beneficial effect of galangin on memory impairment.

Dementia diseases are one of the most common health and socioeconomic problems in the elderly population worldwide (35). Acetylcholinesterase inhibitors are first-line treatment options in Alzheimer's disease and other dementias $(36,37)$. In this study, we compared the effects of galangin with the acetylcholinesterase inhibitor donepezil, which is commonly prescribed in dementia and observed that galangin is comparable to donepezil.

\section{Conclusion}

Galangin may improve memory impairment. Both nicotinic and muscarinic receptors may be involved in this effect. We suggest that galangin may be a potential promising agent in the prevention and treatment of cognitive dysfunction.

\section{References}

1. Atkins ER, Bulsara MK, Panegyres PK. The natural history of earlyonset dementia: the Artemis Project. BMJ Open 2012; 2 (5): e001764.

2. Meng X, D'Arcy C. Education and dementia in the context of the cognitive reserve hypothesis: a systematic review with meta-analyses and qualitative analyses. PLoS One 2012; 7 (6): e38268.

3. Knopman DS. The initial recognition and diagnosis of dementia. Am J Med 1998; 104 (4A): 2S-12S; discussion 39S-42S.

4. Murty VP, LaBar KS, Hamilton DA, Adcock RA. Is all motivation good for learning? Dissociable influences of approach and avoidance motivation in declarative memory. Learn Mem 2011; 18 (11): 712-717.

5. Kumaran D, Hassabis D, Spiers HJ, Kumaran D, Hassabis D, Spiers HJ. Impaired spatial and non-spatial configural learning in patients with hippocampal pathology. Neuropsychologia 2007; 45 (12): 2699-2711.

6. Luchicchi A, Bloem B, Viaña JN, Mansvelder HD, Role LW. Illuminating the role of cholinergic signaling in circuits of attention and emotionally salient behaviors. Front Synaptic Neurosci 2014; 6: 24.

7. D'Hooge R, De Deyn PP. Applications of the Morris water maze in the study of learning and memory. Brain Res Brain Res Rev 2001; 36 (1): 60-90.

8. Scarr E, Gibbons AS, Neo J, Udawela M, Dean B. Cholinergic connectivity: it's implications for psychiatric disorders. Front Cell Neurosci 2013; 7: 55.

9. Tripathi PN, Srivastava P, Sharma $\mathbf{P}$ et al. Biphenyl-3-oxo-1,2,4triazine linked piperazine derivatives as potential cholinesterase inhibitors with anti-oxidant property to improve the learning and memory. Bioorg Chem 2019; 85: 82-96.

10. Ghezzi L, Scarpini E, Galimberti D. Disease-modifying drugs in Alzheimer's disease. Drug Des Devel Ther 2013; 7: 1471-1478.

11. Pohanka M. Inhibitors of acetylcholinesterase and butyrylcholinesterase meet immunity. Int J Mol Sci 2014; 15 (6): 9809-9825.

12. Chambon C, Jatzke C, Wegener N, Gravius A, Danysz W. Using cholinergic $\mathrm{M}(1)$ receptor positive allosteric modulators to improve me- mory via enhancement of brain cholinergic communication. Eur J Pharmacol 2012; 697 (1-3): 73-80.

13. Singh M, Kaur M, Silakari O. Flavones: an important scaffold for medicinal chemistry. Eur J Med Chem 2014; 84: 206-239.

14. Ginter E, Simko V. Plant polyphenols in prevention of heart disease. Bratisl Lek Listy 2012;113 (8): 476-80.

15. Chien ST, Shi MD, Lee YC, Te CC, Shih YW. Galangin, a novel dietary flavonoid, attenuates metastatic feature via PKC/ERK signaling pathway in TPA-treated liver cancer HepG2 cells. Cancer Cell Int 2015; 15: 15.

16. Jung YC, Kim ME, Yoon JH et al. Anti-inflammatory effects of galangin on lipopolysaccharide-activated macrophages via ERK and NF- $\mathrm{kB}$ pathway regulation. Immunopharmacol Immunotoxicol 2014; 36 (6): 426-432.

17. Guo AJ, Xie HQ, Choi RC et al. Galangin, a flavonol derived from Rhizoma Alpiniae Officinarum, inhibits acetylcholinesterase activity in vitro. Chem Biol Interact 2010; 187 (1-3): 246-248.

18. Yau JL, Noble J, Hibberd $\mathbf{C}$ et al. Chronic treatment with the antidepressant amitriptyline prevents impairments in water maze learning in aging rats. J Neurosci 2002; 22 (4): 1436-1442.

19. Vorhees CV, Williams MT. Assessing spatial learning and memory in rodents. ILAR J 2014; 55 (2): 310-332.

20. Hohnadel E, Bouchard K, Terry AV Jr. Galantamine and donepezil attenuate pharmacologically induced deficits in prepulse inhibition in rats. Neuropharmacology 2007; 52: 542-551.

21. Tanyeri P, Buyukokuroglu ME, Mutlu O et al. Effects of ziprasidone, SCH23390 and SB277011 on spatial memory in the Morris water maze test in naive and MK-801 treated mice. Pharmacol Biochem Behav 2015; 138: 142-147.

22. Takizawa C, Thompson PL, van Walsem A, Faure C, Maier WC. Epidemiological and economic burden of Alzheimer's disease: a systematic literature review of data across Europe and the United States of America. J Alzheimers Dis 2015; 43 (4): 1271-1284.

23. Shaikh S, Verma A, Siddiqui S et al. Current acetylcholinesteraseinhibitors: a neuroinformatics perspective. CNS Neurol Disord Drug Targets 2014; 13 (3): 391-401.

24. Liu C, Ma M, Zhang J, Gui S, Zhang X, Xue S. Galangin inhibits human osteosarcoma cells growth by inducing transforming growth factor$\beta 1$-dependent osteogenic differentiation. Biomed Pharmacother 2017; 89: $1415-1421$.

25. Abubakar IB, Malami I, Yahaya Y, Sule SM. A review on the ethnomedicinal uses, phytochemistry and pharmacology of Alpinia officinarum Hance. J Ethnopharmacol 2018; 224: 45-62.

26. Katalinić M, Rusak G, Domaćinović Barović J et al. Structural aspects of flavonoids as inhibitors of human butyrylcholinesterase. Eur J Med Chem 2010; 45 (1): 186-192.

27. Shaikh S,Ahmad SS, Ansari MAet al. Prediction of comparative inhibition efficiency for a novel natural ligand, galangin against human brain acetylcholinesterase, butyrylcholinesterase and 5-lipoxygenase: a neuroinformatics study. CNS Neurol Disord Drug Targets 2014; 13 (3): 452-459.

28. Lian W, Fang J, Xu L et al. DL0410 Ameliorates Memory and Cognitive Impairments Induced by Scopolamine via Increasing Cholinergic Neurotransmission in Mice. Molecules 2017; 22 (3). pii: E410.

29. Skovgård K, Agerskov C, Kohlmeier KA, Herrik KF. The 5-HT receptor antagonist ondansetron potentiates the effects of the acetylcho- 


\section{1-886}

linesterase inhibitor donepezil on neuronal network oscillations in the rat dorsal hippocampus. Neuropharmacology 2018; 143: 130-142.

30. Lei Y, Chen J, Zhang $\mathbf{W}$ et al. In vivo investigation on the potential of galangin, kaempferol and myricetin for protection of D-galactoseinduced cognitive impairment. . Food Chem 2012; 135 (4): 2702-2707.

31. Ionita R, Postu PA, Beppe GJ et al. Cognitive-enhancing and antioxidant activities of the aqueous extract from Markhamia tomentosa (Benth.) K. Schum. stem bark in a rat model of scopolamine. Behav Brain Funct 2017; 13 (1): 5.

32. Baakman AC, Alvarez-Jimenez R, Rissmann R et al. An Anti-Nicotinic Cognitive Challenge Model using Mecamylamine in Comparison with the Anti-Muscarinic Cognitive Challenge using Scopolamine. Br J Clin Pharmacol 2017; 83 (8): 1676-1687.

33. Huang L, Lin M, Zhong X, Yang H, Deng M. Galangin decreases p-tau, $A \beta 42$ and $\beta$-secretase levels, and suppresses autophagy in okadaic acid-induced PC12 cells via an Akt/GSK3 $3 /$ mTOR signaling-dependent mechanism. Mol Med Rep 2019; 19 (3): 1767-1774.
34. Kim ME, Park PR, Na JY, Jung I, Cho JH, Lee JS. Anti-neuroinflammatory effects of galangin in LPS-stimulated BV-2 microglia through regulation of IL- $1 \beta$ production and the NF- $\kappa \mathrm{B}$ signaling pathways. Mol Cell Biochem 2019; 451 (1-2): 145-153.

35. Dreier-Wolfgramm A, Michalowsky B, Austrom MG et al. Dementia care management in primary care : Current collaborative care models and the case for interprofessional education. Z Gerontol Geriatr 2017; 50 (Suppl 2): 68-77.

36. Bakhtiari S, Moghadam NB, Ehsani M, Mortazavi H, Sabour S, Bakhshi M. Can Salivary Acetylcholinesterase be a Diagnostic Biomarker for Alzheimer? J Clin Diagn Res 2017; 11 (1): ZC58-ZC60.

37. Mohammadi-Farani A, Abdi N, Moradi A, Aliabadi A. 2-(2-(4-Benzoylpiperazin-1-yl) ethyl) isoindoline-1,3-dione derivatives: Synthesis, docking and acetylcholinesterase inhibitory evaluation as anti-alzheimer agents. Iran J Basic Med Sci 2017; 20 (1): 59-66.

Received June 20, 2019. Accepted July 28, 2019. 